\title{
Math-Net.Ru
}

Общероссийский математический портал

Т. Я. Азизов, Н. Д. Копачевский, Л. Д. Орлова, Операторный подход к исследованию гидродинамической модели Олдройта, Матем. заметки, 1999, том 65, выпуск 6, 924928

DOI: https://doi.org/10.4213/mzm1128 
Использование Общероссийского математического портала Math-Net.Ru подразумевает, что вы прочитали и согласны с пользовательским соглашением http://www. mathnet.ru/rus/agreement

Параметры загрузки:

IP: 34.227 .88 .159

26 апреля 2023 г., 15:36:08

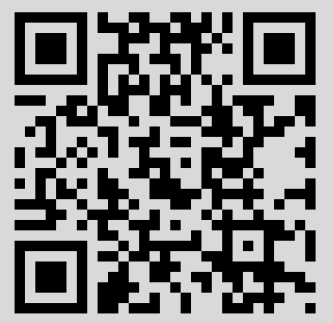




\section{ОПЕРАТОРНЫЙ ПОДХОД К ИССЛЕДОВАНИЮ ГИДРОДИНАМИЧЕСКОЙ МОДЕЛИ ОЛДРОЙТА}

\section{Т. Я. Азизов, Н. Д. Копачевский, Л. Д. Орлова}

1. В сепарабельном гильбертовом пространстве $\mathscr{H}$ рассмотрим задачу Коши для интегродифоференциального уравнения

$$
\frac{d u}{d t}+A_{0} u+\sum_{k=1}^{m} \int_{0}^{t} e^{-\gamma_{k}(t-s)} A_{k} u(s) d s=f(t), \quad u(0)=u^{0}
$$

Здесь $u=u(t)$ - искомая функция со значениями в $\mathscr{H}, \gamma_{k}$ - положительные постоянные, $0<\gamma_{1}<$ $\cdots<\gamma_{m}<\infty ; f(t)$ - заданная функция со значениями в $\mathscr{H}, u^{0} \in \mathscr{H}$. Через $A_{k}(k=0, \ldots, m)$ в (1) обозначены неограниченные самосопряженные положительно определенные операторы с областями определения $\mathscr{D}\left(A_{k}\right)=\mathscr{D}\left(A_{0}\right)$ и компактными обратными $0<A_{k}^{-1} \in \mathfrak{S}_{\infty}(k=0, \ldots, m)$.

Вид задачи (1) и свойства операторных коэффициентов $A_{k}$ позволяют перейти от интегродифференциального уравнения, рассматриваемого в гильбертовом пространстве $\mathscr{H}$, к дифференциальному уравнению первого порядка в пространстве $\widetilde{\mathscr{H}}$, являющемся ортогональной суммой $m+1$ экземпляров пространства $\mathscr{H}$.

Работа первого автора выполнена при частичной поддержке Российского фонда фундаментальных исследований, грант № 96-15-96091. Работа второго и третьего авторов выполнена при частичной поддержке координационного плана № 1 научно-исследовательских работ министерства образования Украины на 1997-99г. 
Пусть $u(t)$ - сильное решение задачи (1). Вводя новые искомые функции $u_{k}(t)(k=0, \ldots, m)$ согласно формулам

$$
u_{0}(t):=u(t), \quad u_{k}(t):=\int_{0}^{t} e^{-\gamma_{k}(t-s)} A_{k}^{1 / 2} u_{0}(s) d s, \quad k=1, \ldots, m,
$$

перейдем к дифференциальному уравнению

$$
\frac{d \widetilde{u}}{d t}+\mathscr{A}_{0} \widetilde{u}=\widetilde{f}(t), \quad \widetilde{u}(0)=\widetilde{u}^{0},
$$

в гильбертовом пространстве

$$
\widetilde{\mathscr{H}}:=\mathscr{H}_{0} \oplus \widehat{\mathscr{H}}_{1}, \quad \mathscr{H}_{0}:=\mathscr{H}, \quad \widehat{\mathscr{H}}_{1}:=\bigoplus_{k=1}^{m} \mathscr{H}_{k}, \quad \mathscr{H}_{k}:=\mathscr{H}, \quad k=1, \ldots, m,
$$

где

$$
\widetilde{u}(t):=\left(u_{0}(t), \widehat{u}_{1}(t)\right)^{t}, \quad \widehat{u}_{1}(t):=\left(u_{1}(t), \ldots, u_{m}(t)\right)^{t}, \quad \widetilde{f}(t):=(f(t), \widehat{0})^{t}, \quad \widetilde{u}(0)=\left(u^{0}, \widehat{0}\right)^{t},
$$

а оператор $\mathscr{A}_{0}$ в ортогональном разложении (3) имеет следующее матричное представление:

$$
\begin{gathered}
\mathscr{A}_{0}:=\left(A_{i j}\right)_{i, j=0}^{1}, \quad A_{00}:=A_{0}, \quad A_{01}:=\left(A_{1}^{1 / 2}, \ldots, A_{m}^{1 / 2}\right) \\
A_{10}:=-\left(A_{1}^{1 / 2}, \ldots, A_{m}^{1 / 2}\right)^{t}, \quad A_{11}:=\operatorname{diag}\left(\gamma_{k} I\right)_{k=1}^{m} .
\end{gathered}
$$

Дальнейшее исследование свойств решений задачи (1) и ассоциированной с ней задачи (2) основано на изучении свойств матричного оператора $\mathscr{A}_{0}$.

Отметим, что оператор $\mathscr{A}_{0}$, заданный формулами $(5)$ на плотном в $\widetilde{\mathscr{H}}$ множестве $\mathscr{D}\left(\mathscr{A}_{0}\right):=$ $\mathscr{D}\left(A_{0}\right) \oplus \widehat{\mathscr{D}}_{1}^{1 / 2}, \widehat{\mathscr{D}}_{1}^{1 / 2}:=\bigoplus_{k=1}^{m} \mathscr{D}\left(A_{k}^{1 / 2}\right), \mathscr{D}\left(A_{k}^{1 / 2}\right)=\mathscr{D}\left(A_{0}^{1 / 2}\right)(k=1, \ldots, m)$, является равномерно аккретивным оператором:

$$
\operatorname{Re}\left(\mathscr{A}_{0} \widetilde{u}, \widetilde{u}\right)_{\widetilde{\mathscr{H}}} \geqslant c\|\widetilde{u}\|_{\widetilde{\mathscr{H}}}^{2}, \quad \widetilde{u} \in \mathscr{D}\left(\mathscr{A}_{0}\right), \quad c=\min \left(\lambda_{1}\left(A_{0}\right), \gamma_{1}\right)>0
$$

где $\lambda_{1}\left(A_{0}\right)$ - минимальное собственное значение оператора $A_{0}$.

Более того, $\mathscr{A}_{0}$ - в существенном максимальный аккретивный оператор, т.е. его замыкание $\mathscr{A}=$ $\overline{\mathscr{A}}_{0}$ - максимальный аккретивный оператор. Введем операторы $Q_{k}:=A_{k}^{1 / 2} A_{0}^{-1 / 2}, \mathscr{D}\left(Q_{k}\right)=$ $\mathscr{H}_{k}=\mathscr{H}_{0}, Q_{k}^{+}:=A_{0}^{-1 / 2} A_{k}^{1 / 2}, \mathscr{D}\left(Q_{k}^{+}\right):=\mathscr{D}\left(A_{k}^{1 / 2}\right)(k=1, \ldots, m)$, а также операторные столбцы и строки $Q_{10}:=\left(Q_{1}, \ldots, Q_{m}\right)^{t}, \mathscr{D}\left(Q_{10}\right)=\mathscr{H}_{0}, Q_{01}^{+}:=\left(Q_{1}^{+}, \ldots, Q_{m}^{+}\right), \mathscr{D}\left(Q_{01}^{+}\right)=\widehat{\mathscr{D}}_{1}^{1 / 2} \subset \widehat{\mathscr{H}}_{1}$. Справедливы соотношения $Q_{k}^{+}=Q_{k}^{*}\left|\mathscr{D}\left(A_{k}^{1 / 2}\right)(k=1, \ldots, m), Q_{01}^{+}=Q_{10}^{*}\right| \widehat{\mathscr{D}}_{1}^{1 / 2}$, причем замыкание по непрерывности оператора $Q_{k}^{+}$совпадает с $Q_{k}^{*}$, а оператора $Q_{01}^{+}-$с $Q_{10}^{*}$.

Теперь мы можем указать область определения оператора $\mathscr{A}$

$$
\mathscr{D}(\mathscr{A})=\left\{\widetilde{u}=\left(u_{0}, \widehat{u}_{1}\right)^{t} \in \widetilde{\mathscr{H}}: u_{0}+A_{0}^{-1 / 2} Q_{10}^{*} \widehat{u}_{1} \in \mathscr{D}\left(A_{0}\right)\right\}
$$

и его действие

$$
\mathscr{A} \widetilde{u}=\left(A_{0}\left(u_{0}+A_{0}^{-1 / 2} Q_{10}^{*} \widehat{u}_{1}\right)\right) \oplus\left(-Q_{10} A_{0}^{1 / 2} u_{0}+A_{11} \widehat{u}_{1}\right), \quad \widetilde{u} \in \mathscr{D}(\mathscr{A}) .
$$

Указанные выше свойства матричного оператора $\mathscr{A}$ позволяют свести проблему (1) к хорошо изученной задаче для дифференциального уравнения первого порядка с максимальньм диссипативным операторньм коэффициентом. 
Tеорема 1. Пусть в задаче (1) выполнены условия $u_{0} \in \mathscr{D}\left(A_{0}\right), f(t) \in C^{1}[0, T ; \mathscr{H}]$. Тогда эта задача имеет (единственное) сильное решение на $[0, T]$.

Доказательство основано на рассмотрении задачи Коши

$$
\frac{d \widetilde{u}}{d t}+\mathscr{A} \widetilde{u}=\widetilde{f}(t), \quad \widetilde{u}(0)=\widetilde{u}^{0},
$$

и проверке существования и единственности ее решения, если $\widetilde{u}(t), \widetilde{f}(t)$ и $\widetilde{u}(0)$ выражаются с помощью формул (4).

ЗАмеч ание 1. Условие непрерьвной дифференцируемости функции $f(t)$ в формулировке теоремы 1 можно заменить любым другим, обеспечиваюшим сильную разрешимость задачи (6). В частности, можно положить $f(t) \in C[0, T ; \mathscr{H}], f(t) \in \mathscr{D}\left(A_{0}\right), A_{0} f(t) \in C[0, T ; \mathscr{H}]$.

2. Нормальными колебаниями называют решения однородной задачи (6), зависящие от $t$ по закону $\widetilde{u}(t)=\widetilde{u} e^{-\lambda t}, \widetilde{u} \in \widetilde{\mathscr{H}}$. Подставляя эти функции в однородное уравнение (6), приходим для определения амплитудных элементов $\widetilde{u} \in \widetilde{\mathscr{H}}$ и собственных значений $\lambda \in \mathbb{C}$ к спектральной задаче

$$
\mathscr{A} \widetilde{u}=\lambda \widetilde{u}, \quad \widetilde{u} \in \mathscr{D}(\mathscr{A}),
$$

ассоциированной с исходной эволюционной задачей (1).

Теорема 2. Задача (7) имеет счетное множество положительных собственных значений $\left\{\lambda_{n}^{(\infty)}\right\}_{n=1}^{\infty}$ с единственной предельной точкой $\lambda=+\infty$ и собственными элемен$\operatorname{maмu}\left\{\widetilde{u}_{n}^{(\infty)}\right\}_{n=1}^{\infty}, \widetilde{u}_{n}^{(\infty)}=\left(u_{0 n}^{(\infty)}, \widehat{u}_{1 n}^{(\infty)}\right)^{t} \in \mathscr{H}_{0} \oplus \widehat{\mathscr{H}}_{1}=\widetilde{\mathscr{H}}$, проекu, uи $\left\{u_{0 n}^{(\infty)}\right\}_{n=1}^{\infty}$ которьх на $\mathscr{H}_{0}=\mathscr{H}$ образуют базис Рисса с конечным дефектом в пространстве $\mathscr{H}$. Если ви-

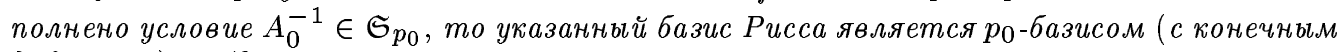
дефектом) в $\mathscr{H}$.

Пусть в задаче $(7)$ операторы $A_{k}(k=1, \ldots, m)$, порождающие оператор $\mathscr{A}$, удовлетворяют условиям

$$
A_{k}=\alpha_{k} A_{0}, \quad \alpha_{k}>0, \quad k=1, \ldots, m .
$$

Этот простой, но важный частный случай позволяет провести исследование спектральной задачи (7) достаточно полно. Однако, мы рассмотрим здесь более общую ситуацию, когда результат "почти" такой же:

$$
Q_{k}^{*} Q_{k}=\alpha_{k} I+F_{k}, \quad F_{k}=F_{k}^{*} \in \mathfrak{S}_{\infty} .
$$

Теорема 3. Если выполнены условия (9), то спектр оператора $\mathscr{A}$ дискретен, за исключением $\infty$ и тонечных точек сгущения $\left\{\beta_{k}\right\}_{k=1}^{m}$, являющихся нулями функции

$$
e_{0}(\lambda):=1+\sum_{k=1}^{m} \frac{\alpha_{k}}{\gamma_{k}-\lambda}
$$

и удовлетворяющих условию $0<\gamma_{1}<\beta_{1}<\cdots<\gamma_{m}<\beta_{m}<\infty$. При этом $\sigma(\mathscr{A}) \backslash(\{\infty\} \cup$ $\left.\left\{\beta_{k}\right\}_{k=1}^{m}\right)$ состоит не более чем из конечного мнохества невещественных собственных значений и $m+1$ ветвей положительных собственных значений, причем одна из ветвей $\left\{\lambda_{k}^{(\infty)}\right\}_{k=1}^{\infty}$ стремится $\kappa+\infty$, а другие $m$ ветвей $\left\{\left\{\lambda_{k}^{(j)}\right\}_{k=1}^{\infty}\right\}_{j=1}^{m}-\kappa\left\{\beta_{k}\right\}_{k=1}^{m}=\sigma_{\mathrm{ess}}(\mathscr{A}) \backslash$ $\{+\infty\}$ соответственно.

$\Pi$ Пи этом если $\left\{\widetilde{u}_{n}^{(\infty)}\right\}_{n=1}^{\infty}, \widetilde{u}_{n}^{(\infty)}=\left(u_{0 n}^{(\infty)}, \widehat{u}_{1 n}^{(\infty)}\right)^{t} \in \mathscr{H}_{0} \oplus \widehat{\mathscr{H}}_{1}=\widetilde{\mathscr{H}}($ coответственно $\left.\left\{\widetilde{u}_{n}^{(j)}\right\}_{n=1}^{\infty}, \widetilde{u}_{n}^{(j)}=\left(u_{0 n}^{(j)}, \widehat{u}_{1 n}^{(j)}\right)^{t} \in \mathscr{H}_{0} \oplus \widehat{\mathscr{H}}_{1}=\widetilde{\mathscr{H}}\right),-$ собственные элементы оператора $\mathscr{A}$, отвечающие ветви $\left\{\lambda_{k}^{(\infty)}\right\}_{k=1}^{\infty}$ собственных значений, стремящ,ейся $\kappa+\infty$ (соответственно ветвям $\left\{\lambda_{k}^{(j)}\right\}_{k=1}^{\infty}$, стремящимся $\left.\kappa\left\{\beta_{j}\right\}, j=1, \ldots, m\right)$, то их проекции $\left\{u_{0 n}^{(\infty)}\right\}_{n=1}^{\infty}$ на $\mathscr{H}_{0}=\mathscr{H}$ образуют базис Рисса с конечным дефектом в пространстве $\mathscr{H}$ 
(соответственно замкнутая линейная оболочка проекций $\left\{\widehat{u}_{1 n}^{(j)}\right\}_{n=1}^{\infty}, j=1, \ldots, m$, на $\widehat{\mathscr{H}}_{1}$ - подпространство конечной коразмерности - в $\left.\widehat{\mathscr{H}}_{1}\right)$.

При дополнительном условии

$$
\operatorname{Ker}\left(\mathscr{A}-\beta_{j} \mathscr{I}\right)=\{0\}, \quad j=1, \ldots, m,
$$

система $\left\{\widehat{u}_{1 n}^{(j)}\right\}_{n=1}^{\infty}, j=1, \ldots, m$, образует базис Рисса в своей замкнутой линейной обоnочке.

Ecлu $A^{\prime}\left(\beta_{j}\right) \gg 0, j=1, \ldots, m$, əде

$$
A(\lambda):=e_{0}(\lambda) I+\sum_{k=1}^{m}\left(\gamma_{k}-\lambda\right)^{-1} F_{k}-\lambda A_{0}^{-1}
$$

то (без предположения (10)) проекиии векторов $\left\{\widehat{u}_{1 n}^{(j)}\right\}_{n=1}^{\infty}$ на $\mathscr{H}_{j}, j=1, \ldots$, m, образуют базис Рисса с конечным дефектом в $\mathscr{H}_{j}=\mathscr{H}, j=1, \ldots, m$.

Отметим, что при условии (8) автоматически выполнено и условие (10).

3. В качестве приложения проведенного исследования можно рассмотреть задачу о малых движениях вязкоупругой жидкости, заполняющей произвольную область $\Omega \subset \mathbb{R}^{3}$ (см., например, [1]). Эта задача состоит в нахождении поля скоростей $\vec{u}(t, x)$ и поля давлений $p(t, x)$ из системы уравнений с краевым и начальным условиями:

$$
\begin{gathered}
\frac{\partial \vec{u}}{\partial t}=-\rho^{-1} \nabla p+\nu I_{0}(t)(\Delta \vec{u})+\vec{f}, \quad \operatorname{div} \vec{u}=0 \text { в } \Omega, \\
\left(I_{0}(t) \vec{v}\right)(t, x):=\vec{v}(t, x)+\sum_{j=1}^{m} \alpha_{j} \int_{0}^{t} e^{-\gamma_{j}(t-s)} \vec{v}(s, x) d s \\
\vec{u}=\overrightarrow{0} \text { на } S=\partial \Omega, \quad \vec{u}(0, x)=\vec{u}^{0}(x),
\end{gathered}
$$

где $\nu>0$ и $\rho>0$ - коэффициент кинематической вязкости и плотность жидкости соответственно, $\alpha_{j}>0(j=1, \ldots, m), 0<\gamma_{1}<\cdots<\gamma_{m}$, a $\vec{f}=\vec{f}(t, x)$ - плотность малого поля массовых сил, наложенного на гравитационное поле. Для перехода от (11) к задаче вида (1) будем считать функции $\vec{u}(t, x)$ и $\nabla p(t, x)$ при каждом $t$ элементами гильбертова пространства вектор-функций $\vec{L}_{2}(\Omega)$.

Воспользуемся ортогональным разложением

$$
\begin{gathered}
\vec{L}_{2}(\Omega)=\vec{J}_{0}(\Omega) \oplus \vec{G}(\Omega), \quad \vec{G}(\Omega):=\left\{\vec{v} \in \vec{L}_{2}(\Omega): \vec{v}=\nabla p\right\}, \\
\vec{J}_{0}(\Omega):=\left\{\vec{u} \in \vec{L}_{2}(\Omega): \operatorname{div} \vec{u}=0 \text { в } \Omega, u_{n}:=\vec{u} \cdot \vec{n}=0 \text { на } \partial \Omega\right\},
\end{gathered}
$$

где $\vec{n}$ - вектор единичной нормали к $\partial \Omega, \operatorname{a~} \operatorname{div} \vec{u}$ и $u_{n}$ понимаются как обобщенные функции конечного порядка (см., например, $[2, \S 2.1])$. Тогда в силу уравнений $(11)$ имеем $\vec{u}(t, x) \in \vec{J}_{0}(\Omega)$, $\nabla p(t, x) \in \vec{G}(\Omega)$.

Пусть $P_{0}$ - ортопроектор на $\vec{J}_{0}(\Omega)$, а $P_{G}$-ортопроектор на $\vec{G}(\Omega), P_{0}+P_{G}=I$. Считая функции $\vec{u}(t, x)$ и $\nabla p(t, x)$ классическим решением задачи (11) и применяя ортопроекторы $P_{0}$ и $P_{G}$ к обеим частям первого уравнения (11), получим

$$
\begin{gathered}
\frac{d \vec{u}}{d t}+\nu I_{0}(t)\left(A_{0} \vec{u}\right)=\vec{f}_{0}(t), \quad \vec{u}(0)=\vec{u}^{0}, \\
A_{0} \vec{u}:=-P_{0}(\Delta \vec{u}), \quad \vec{f}_{0}:=P_{0} \vec{f}, \quad \rho^{-1} \nabla p=\nu I_{0}(t) P_{G}(\Delta \vec{u})+P_{G} \vec{f} .
\end{gathered}
$$

Пусть граница $\partial \Omega$ области $\Omega$ является дважды непрерьвно дифференцируемой $\left(\partial \Omega \in C^{2}\right)$. Тогда, как известно [3],

$$
\mathscr{D}\left(A_{0}\right)=\vec{J}_{0}^{2}(\Omega):=\left\{\vec{u} \in \vec{J}_{0}(\Omega): \Delta \vec{u} \in \vec{L}_{2}(\Omega), \vec{u}=\overrightarrow{0} \text { на } \partial \Omega\right\} .
$$


Отметим также, что оператор $A_{0}$ положительно определен в $\vec{J}_{0}(\Omega)$, причем обратный оператор компактен: $0<A_{0}^{-1} \in \mathfrak{S}_{\infty}$.

Задаче (12) при $m \geqslant 2$ отвечает обобщенная модель Олдройта вязкоупругой жидкости [4]-[8]; при $m=1$ получаем модель Олдройта (см. [1]). Очевидно, уравнение (12) является частным случаем задачи $(1)$ в пространстве $\mathscr{H}=\vec{J}_{0}(\Omega)$, при этом следует заменить $A_{0}$ на $\nu A_{0}$, а $A_{k}$ на $\nu \alpha_{k} A_{0}$ $(k=1, \ldots, m)$, где $A_{0}$ - оператор Стокса (13), (14).

Таким образом, мы находимся в условиях п. 2, значит для этой гидродинамический задачи справедливы все полученные в нем результаты.

\section{СПИСОК ЦИТИРОВАННОЙ ЛИТЕРАТУРЫ}

1. Эйрих Ф. (ред. ) Реология. Теория и приложения. М.: ИЛ, 1962. 2. Копачевский Н. Д., Крейн С. Г., Нго Зуй Кан. Операторные методы в линейной гидродинамике. Эволюционные и спектральные задачи. М.: Наука, 1989. 3. Л адыженская О. А. Математические вопросы динамики вязкой несжимаемой жидкости. М.: Наука, 1970. 4. Милославский А. И. // УМН. 1986. Т. 41. №1. С. 199-200. 5. Милославский А. И. // Докл. АН СССР. 1989. Т. 309. № 3. С. 532-536. 6. Милославский А. И. // XIV пкола по теории операторов в функциональных пространствах. Тезисы докл. Новгород: Изд. Новгородского ГПИ, 1989. 7. Милославский А. И. // Деп. УкрНИИНТИ 22. 05. 89.. № 1221. 8. Милославский А. И. // Деп. УкрНИИНТИ 22. 05. 89.. № 1321.

Воронежский государственный университет

Поступило

Симферопольский государственный университет

02.09 .98

E-mail: azizov@tom.vsu.ru 